\title{
Siewert solutions of transcendental equations, generalized Lambert functions and physical applications
}

https://doi.org/10.1515/phys-2018-0034

Received July 27, 2017; accepted November 25, 2017

\begin{abstract}
Several classes of transcendental equations, mainly eigenvalue equations associated to non-relativistic quantum mechanical problems, are analyzed. Siewert's systematic approach of such equations is discussed from the perspective of the new results recently obtained in the theory of generalized Lambert functions and of algebraic approximations of various special or elementary functions. Combining exact and approximate analytical methods, quite precise analytical outputs are obtained for apparently untractable problems. The results can be applied in quantum and classical mechanics, magnetism, elasticity, solar energy conversion, etc.
\end{abstract}

Keywords: transcendental equations; generalized Lambert functions; Langevin function; Currie-Weiss theory; algebraic approximations

PACS: $02.30 . \mathrm{Gp} ; 02.30 . \mathrm{Mv}$; 02.60.Cb

\section{Introduction}

Obtaining exact solutions for the equations of physics is a fascinating endeavour for scientists. However, this goal can rarely be achieved. In quantum mechanics, for instance, there are very few cases when, for a given problem, both the eigenfunctions and the eigenvalues can be obtained exactly. This happens when the Hamiltonian has some symmetry properties, or when it is extremely simple. But otherwise, even for the most elementary onedimensional potentials, like the square well potential, given by [1]:

\footnotetext{
*Corresponding Author: Victor Barsan: National Institute of Physics and Nuclear Engineering (NIPNE), Str. Reactorului 30, 077125 Magurele and UNESCO Chair at HHF, Str. Atomistilor 407, 077125 Magurele, Romania, E-mail: vbarsan@theory.nipne.ro
}

$$
V(x)=\left\{\begin{array}{cc}
-V_{0}, & -\frac{a}{2}<x<\frac{a}{2} \\
0, & \text { otherwise }
\end{array}\right.
$$

with $V_{0}>0$, when the wave functions can be expressed in terms of elementary functions, the eigenvalues of the energy $E$ (or of the wave vector $k=\sqrt{2 m|E|}$ ) cannot be obtained exactly. Namely, the eigenvalues of the wave vector $k$ are given by a transcendental equation:

$$
\frac{\sin k a}{k a}=p
$$

where $p$ is a dimensionless parameter, describing both the potential and the particle, and $a$ is the length of the well. The solutions of (2) are

$$
k=\frac{1}{a} \xi(p)
$$

with $\xi(p)$ satisfying the equation:

$$
\frac{\sin \xi(p)}{\xi(p)}=p
$$

Evidently, $\xi(p)$ cannot be written as a finite combination of elementary functions. Various methods exact or approximate - of solving eq. (2) - (4) have been recently discussed in [2-5].

What is currently called "to solve the Schroedinger equations for a square well", means actually to solve a linear Sturm - Liouville problem, i.e. to obtain the solutions of a linear differential equation - the Schroedinger equation - which satisfy some boundary conditions. In the previously mentioned case, the solutions $\psi(x)$ must vanish in the limit $x \rightarrow \infty$. In other cases, for instance in the linear theory of elasticity applied to dipolar materials with voids [6], or in the non-classical theories of thermoelasticity [7], [8], more general boundary conditions are imposed.

Similarly, the exact expressions of eigenvalues of energy (or wave vectors) of other quantum mechanical problems are the solutions of more or less similar transcendental equations (see for instance [9]):

$$
\begin{aligned}
\frac{\tan \xi(p)}{\xi(p)} & =p, \xi(p) \tan \xi(p)=p, \frac{\tanh \xi(p)}{\xi(p)} \\
& =p, \xi(p) \tanh \xi(p)=p
\end{aligned}
$$


to quote the simplest examples only. In more complicated cases, instead of $p$, the r.h.s. of equations similar to (4) contains polynomials or rational functions of $p$, etc. Such equations do not appear in quantum mechanics only, but also in electromagnetism, fluid dynamics, elasticity, etc.

There are few systematic approaches of transcendental equations like (3) and (4). One of them has been initiated by Stewart and his co-workers, some 40 years ago, using the theory of Riemann problems. Another one, developing the concept of generalized Lambert functions, was recently proposed by Mezö, Baricz and Mugnaini, and is subject of intense research.

Meantime, in order to obtain results which are not just numerical, but still preserve a dependence on the physical parameters of the problem, several analytic approximations for the solutions of the transcendental equations have been obtained, in the last decades. Also, mathematical symbolic computation programs, like Mathematica, provide a powerful tool to investigate, both numerically and, in some extent, analytically, the solutions of transcendental equations similar to (4) and (5).

As the exact results are obtained by mathematicians who are not much interested in approximations, and the analytical approximations are obtained by physicists who are not much interested in exact (and sometimes very complicated) solutions, the exchange of information between researchers working in these fields is sometimes deficient. A better communication between scientists involved in these fields could be beneficial for finding new physical applications of mathematical results, semi-quantitative behavior of solutions whose exact form cannot be obtained, etc.

In this paper, we shall make an inventory of Siewert's exact results on transcendental equations, and we shall also mention the approximate analytical results, obtained by other researchers, in the study of these equations. We shall show how a combination of both approaches - the exact and approximate one - can be used for a better understanding of mathematical and physical aspects of the problems under examination.

The structure of this paper is the following. As Siewert's approach is the most comprehensive systematic approach of the transcendental equations, we shall use the series of his papers as the spinal column of our article. A short exposition of his results will be done in Section 2, where an introduction of the generalized Lambert functions is also presented. The first specific application is devoted to the Langevin function, discussed in Section 3; inter alia, an analytic approximation of the inverse Langevin function will be used in order to provide an approximate expression of a certain generalized Lambert func- tion. Such approximations are useful not only in para- or super-paramagnetism, but also in polymer physics and in solar energy conversion.

In Section 4, equations involving hyperbolic and algebraic functions are studied, with applications to the Currie - Weiss theory of ferromagnetism. In Section 5, an approximate solution of an equation involving trigonometric and exponential functions, with applications into the theory of vibrating plates and quantum mechanics, is written in terms of Lambert generalized function, using an algebraic approximation of $\tan x$. In Section 6, both the onedimensional movement of a point electric charge in the field of another, fixed charge, and the Kepler equation for hyperbolic orbits, are analyzed in terms of the Wright $\omega$ function. In Section 7, we shortly discuss two new applications of the Lambert functions in physics. In Section 8, two equations involving algebraic and trigonometric functions - one of them, pertaining to reactor physics, and the other one, to celestial mechanics (Kepler equation for elliptic orbits) are solved, using algebraic approximations of trigonometric functions. Section 9 is devoted to conclusions.

\section{Siewert's approach to transcendental equations and generalized Lambert functions}

In a series of papers published between 1972 and 1976 [10] /S50, [11] /S52, [12] /S57, [13] /S53, [14] /S56, [15] /S59, [16] /S62, [17]/S63, [18] /S68, [19]/S71, [20]/S80, [21] /S89, [22] /S100, [23] /S108, Siewert and his co-workers - Burniston (for [10], [11], [13], [15], [16], [19], [20]), Phelps III (for [22], [23]), Essig (for [14]), Dogget (for [19]) and Burkart (for [17]) studied the solutions of several transcendental equations, important for their physical applications. All the aforementioned publications are available, with open access, on Siewert's web page [24]; the symbols /S50, /S52, etc., in the first three lines of this paragraph, indicate the number of the respective paper in Siewert's publication list, on his web. The approach used in these papers is based "on complex variable analysis and requires ultimately a canonical solution of a certain Riemann problem; the solution of the suitably posed Riemann problem follows immediately from the work of Muskhelishvili [25]", as stated in [11]. The effort invested in this vast research is impressive, and the results are a pioneering and extremely valuable contribution to the development of the theory of transcendental equations. In the same time, the solutions obtained in this 
way are, in general, very complicated and difficult to use in physical applications.

Recently, the interest for these solutions increased, as some of them can be expressed in terms of generalized Lambert functions, and put in a much more usable form, according to the results obtained by Mezö, Baricz [26] and Mugnaini [27]. Similar to the Lambert function $W(a)$, which is defined as the solution of the transcendental equation:

$$
x e^{x}=a
$$

the generalized Lambert function $W\left(t_{1}, t_{2}, \ldots t_{n} ; s_{1}, s_{2}, \ldots s_{m} ; a\right)$ is the solution of a similar, but more complicated transcendental equation [26]:

$$
e^{x} \frac{\left(x-t_{1}\right) \ldots\left(x-t_{n}\right)}{\left(x-s_{1}\right) \ldots\left(x-s_{m}\right)}=a
$$

The parameters $t_{1}, t_{2}, \ldots$ are called upper parameters, and $s_{1}, s_{2}, \ldots$ - lower parameters.

If the denominator, respectively the nominator of the fraction in eq. (7) is equal to 1 - in other words, if the upper, respectively the lower parameters are absent - we shall denote the corresponding solutions $W\left(-; s_{1}, s_{2}, \ldots s_{m} ; a\right)$, respectively $W\left(t_{1}, t_{2}, \ldots t_{n} ;-; a\right)$.

However, as we shall see, not all the Siewert solutions can be expressed in therms of generalized Lambert functions.

The theory of these functions may seem (and effectively be) very complicated, but, as Corless [47] notices, "the Lambert $W$ function is the simplest example of a root of an exponential polynomial", i.e. of an expression of the form:

$$
\sum_{i=1}^{m} P_{i}(x) e^{w_{i} x}
$$

with $P_{i}(x)$ - polynomials in $x$, "and exponential polynomials are the next simplest class of functions after polynomials." The generalized Lambert functions are the roots of an exponential polynomial (8) corresponding to the case $w_{1}=0, w_{2} \neq 0, P_{1} \neq 0, P_{2} \neq 0$ and all $P_{i} \equiv 0$ for $i>2$. The cases already investigated correspond to the situation when both $P_{1}$ and $P_{2}$ have only real roots. Exponential polynomials similar to (8) may appear in the solutions of non-linear Sturm - Liouville problems (see, for instance, [48], eq. (67), where the parameters $w_{i}$ are imaginary) or non-linear fractional Klein - Gordon equation (see, for instance, [49], eq. (23), where the parameters $w_{i}$ are real). So, with the study of the generalized Lambert functions, we assist - and we are free to contribute - to a very important development of mathematics.
Till now, there are two cases when the generalized Lambert function can be put in a convenient form:

(1) If, in eq. (6), $n=1$ and $m=1$, so it contains only one upper parameter, $t_{1}=t$ and one lower parameter, $s_{1}=$ $s, t \neq s$, its solution can be written as series expansion around $a=0$ :

$$
W(t ; s ; a)=t-(t-s) \sum_{n=1}^{\infty} \frac{L_{n}^{\prime}(n(t-s))}{n} e^{-n t} a^{n}, \quad t-s \neq 0
$$

where $L_{n}^{\prime}$ is the derivative of the Laguerre polynomial. The inverses of the functions $\operatorname{coth} x-\frac{1}{x}$ (the Langevin function), $(\tanh x) / x$ and $x \tanh x$ can be expressed as generalized Lambert functions $W(t ; s ; a)$ [26].

(2) If, in eq. (6), $n=2$ and $m=0$, so it contains only two upper parameters $t_{1}$ and $t_{2}$, its solution can be written as a series expansion around $a=0$ :

$$
\begin{aligned}
& W\left(t_{1}, t_{2} ;-; a\right)= \\
& t_{1}-\sum_{n=1}^{\infty} \frac{1}{n \cdot n !}\left(\frac{a n e^{-t_{1}}}{t_{2}-t_{1}}\right)^{n} B_{n-1}\left(-\frac{2}{n\left(t_{2}-t_{1}\right)}\right)
\end{aligned}
$$

where $B_{n}$ are Bessel polynomials.

The generalized Lambert functions represent a field of intense research; for instance, recently Mezö [28] obtained the main properties of $W(t ; s ; a)$, when the parameters $t, s, a$ are complex. We can expect that, in near future, equations similar to (9) and (10) will be obtained for other types of generalized Lambert function, besides $W(t ; s ; a)$ and $W\left(t_{1}, t_{2} ;-; a\right)$

The applications of the generalized Lambert functions $W(t ; s ; a)$ and $W\left(t_{1}, t_{2} ;-; a\right)$ to various physical problems of quantum mechanics, magnetism or hydrodynamics were presented in [29], [30] and [9]

In the next section, we shall start our discussion concerning the Siewert solutions and generalized Lambert functions with the examination of transcendental equations involving the Langevin function.

\section{Transcendental equations involving the Langevin function}

In [20], Siewert and Burniston obtained an exact analytical solution of the equation:

$$
x \operatorname{coth} x=\alpha x^{2}+1
$$

It can be written in terms of the Langevin function

$$
L(x)=\operatorname{coth} x-\frac{1}{x}
$$


as:

$$
L(x)=\alpha x
$$

So, in [20], Siewert and Burniston obtained explicitly the function $x(\alpha)$, or, equivalently, the inverse of the function $L(x) / x$. Mezö and Baritz had obtained the inverse of the Langevin function as a generalized Lambert function with one upper and one lower parameter, $W(t ; s ; a)$. Actually, if $L(x)=a$, the function $L^{-1}(a)$ is:

$$
L^{-1}(a)=-2 W\left(\frac{2}{a+1} ; \frac{2}{a-1} ; \frac{a-1}{a+1}\right)
$$

according to [29], [30], [9].

It is easy to see that, if $0<x<\infty$, then:

$$
0<L(x)<1
$$

and:

$$
0<\frac{L(x)}{x}<\frac{1}{3}
$$

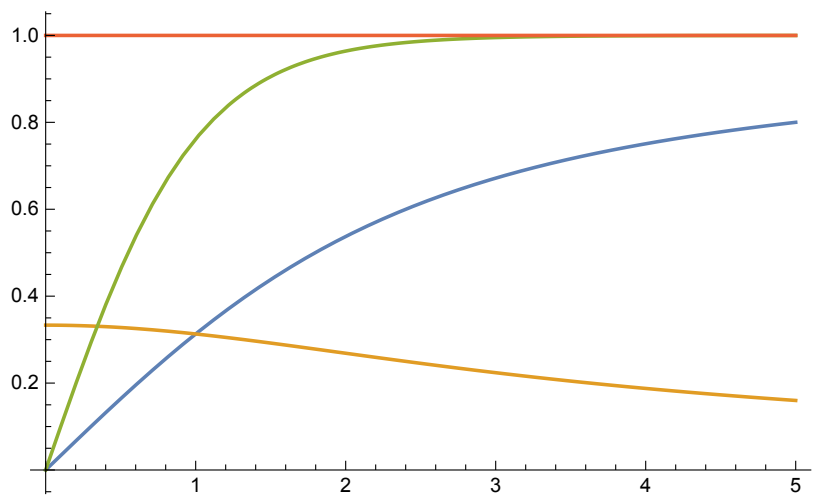

Figure 1: The plot of $\mathrm{L}(\mathrm{x})$ (solid, black), $\tanh (\mathrm{x})$ (purple), $\mathrm{L}(\mathrm{x}) / \mathrm{x}$ (red) and of the constant function $y(x)=1$ (dotted).

It is a simple exercise to check that the solution of (13), so the inverse of the function $L(x) / x$ is a $W\left(t_{1}, t_{2} ; s_{1}, s_{2} ; a\right)$ generalized Lambert function, namely:

$$
\begin{aligned}
& x(\alpha)=\frac{1}{2} W\left(\frac{1+\sqrt{1-4 \alpha}}{\alpha}, \frac{1-\sqrt{1-4 \alpha}}{\alpha} ;\right. \\
& \left.-\frac{1+\sqrt{1-4 \alpha}}{\alpha},-\frac{1-\sqrt{1-4 \alpha}}{\alpha} ; 1\right)
\end{aligned}
$$

So, the inverse of the Langevin function $L(x)$ is a generalized Lambert function with one upper and one lower parameter, and the inverse of $L(x) / x$ is a generalized Lambert function with two upper and two lower parameters. However, as there is no explicit formula for the dependence of $W\left(t_{1}, t_{2} ; s_{1}, s_{2} ; a\right)$ of its parameter, eq. (17) seems to be of limited practical value.
Let us recall now the main applications of the Langevin function in physics. It has been firstly introduced in the context of classical theory of paramagnetism, where it gives the magnetization $M$ as a function of the external magnetic field $H$ and temperature $T$ :

$$
M=n \mu L\left(\frac{\mu H}{k_{B} T}\right)
$$

(see for instance [31], eq. (9.2)). Actually, this is the equation of state for a classical paramagnet. The same formula is valid for superparamagnetic nanoparticles (particles which are small enough to contain one single magnetic domain), at high enough values of temperature $T$ [32], [33].

For physicists, it is important that the Langevin function is a particular case of a function which has a key role in the mean field theory of magnetism, the Brillouin function $B_{S}$, defined as:

$$
B_{S}(x)=\frac{2 S+1}{2 S} \operatorname{coth}\left(\frac{2 S+1}{2 S} x\right)-\frac{1}{2 S} \operatorname{coth}\left(\frac{1}{2 S} x\right)
$$

Indeed,

$$
B_{\infty}(x)=L(x)
$$

Another important particular case corresponds to the halfinteger spin, $S=1 / 2$, when

$$
B_{1 / 2}(x)=\tanh x
$$

The Langevin function and its inverse are relevant not only for magnetism, but also for other domains of physics with important practical applications, as polymers (polymer deformation and flow) [34], [36], [37] or rubber elasticity [35]. An idealized model for rubber-like chain is given in Kubo's treatise of statistical mechanics [38], Problem 17, Ch. 2, p. 135, and is illustrative for understanding how the Langevin function is used in this domain. Another application of the Langevin function pertains to solar energy conversion (daily clearness index) [40]. Actually, Keady noticed that the average of the daily clearness index can be expressed in terms of a Langevin function [39]. Researchers in these fields proposed a large number of useful analytical approximations for $L(x)$ and $L^{-1}(x)$. We shall give an example.

Taking the inverse Langevin function in both sides of (13), we get:

$$
L^{-1}(L(x))=L^{-1}(\alpha x)=\chi
$$


which also determines the function $x(\alpha)$, as eq. (13) or (11) do. Let us use, for $L^{-1}(x)$, the very simple and precise approximation proposed by Kröger, see eq. (10) of [35]:

$$
L^{-1}(x)=\frac{3 x}{\left(1-x^{2}\right)\left(1+0.5 x^{2}\right)}
$$

In this case, the transcendental equation

$$
L^{-1}(\alpha x)=x
$$

has an approximate, but precise and simple algebraic equation, whose physically convenient root is:

$$
x(\alpha)=\frac{1}{\alpha} \sqrt{\frac{\sqrt{3(3-8 \alpha)}-1}{2}}
$$

The identity

$$
f(\alpha)=\frac{x(\alpha) \operatorname{coth} x(\alpha)}{\alpha x(\alpha)^{2}+1}=1
$$

where $x(\alpha)$ is replaced with the approximate solution (25), is fulfilled with a relative error less than 0.003 , as we can see in the plot of Figure 2.

So, we have the approximate relation:

$$
\begin{aligned}
& W\left(\frac{1+\sqrt{1-4 \alpha}}{\alpha}, \frac{1-\sqrt{1-4 \alpha}}{\alpha} ;\right. \\
& \left.-\frac{1+\sqrt{1-4 \alpha}}{\alpha},-\frac{1-\sqrt{1-4 \alpha}}{\alpha} ; 1\right) \\
& \simeq \frac{1}{\alpha} \sqrt{2(\sqrt{3(3-8 \alpha)}-1)}
\end{aligned}
$$

Also, with (14) and (23):

$$
W\left(\frac{2}{a+1} ; \frac{2}{a-1} ; \frac{a-1}{a+1}\right) \simeq-\frac{3}{2} \frac{a}{\left(1-a^{2}\right)\left(1+0.5 a^{2}\right)}
$$

In conclusion, using a precise analytical approximation for the inverse Langevin function (23), we obtained precise approximate analytical expressions for particular cases of generalized Lambert functions, (27) and (28).

Kruger's formula (23) is not the only very precise approximation of the inverse Langevin function; Keady [39] proposed the following one:

$$
L^{-1}(x) \simeq \frac{6}{\pi} \tan \left(\frac{\pi}{2} x\right) \frac{1+0.41770 \tan ^{2}\left(\frac{\pi}{2} x\right)}{1+0.50786 \tan ^{2}\left(\frac{\pi}{2} x\right)}
$$

which is even more precise, as we can see in Figure 2, but not useful for obtaining the inverse of $L(x) / x$. Actually, the error of Keady's approximation is essentially the error of the identity

$$
g(x)=\frac{1}{x} L\left(L^{-1}(x)\right)=1
$$

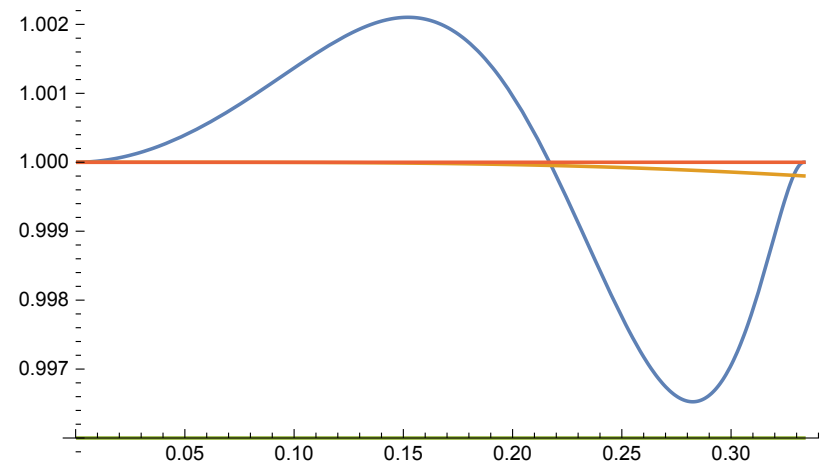

Figure 2: The plot of I.h.s. of the approximate identities (26) - blue, and (30) - brown, and of the constant functions 1 - red, and 0.996 green.

with $L^{-1}(x)$ given by (27). The difference between the form of $f(x)$ and $g(x)$ is due to the difference between the arguments of the Langevin functions in (22) and (27).

The usefulness of Kroeger's formula for putting the equation of state (18) in a more convenient form becomes more clear if we follow Arrot's approach [41].

\section{Equations involving hyperbolic and algebraic functions}

In [17], Siewert and Burkart studied the double zeros of the equation:

$$
x=\tanh (a x+b)
$$

The physical significance of this equation is to be found in the Curie - Weiss theory of magnetism, where the magnetization $M$ can be related to the magnetic field $H$, the spin $S$ and the temperature $T$ by the equation of state:

$$
M=M_{0} B_{S}\left(\frac{g \mu_{B}}{T}(H+\lambda M)\right)
$$

(see [42], eq. (6.14)), where $g$ is the Lande factor, $\mu_{B}$ - the Bohr magneton and $\lambda$ - the molecular field parameter. $B_{S}$ is the Brillouin function, defined in the previous section. If $S=1 / 2$, the Brillouin function becomes the hyperbolic tangent, and eq. (30) coincides with (29). For a very useful discussion of the physical significance of eq. (26), see the excellent study of the Curie - Weiss magnet [44], eq. (22).

If $b=0$, one obtains a numerical value for $a$, namely $a=1$, equivalent to the determination of the Curie temperature (see for instance [42], eq. (6.15) or [43], Ch. 15, eq. (8)). 
Geometrically, a double zero of (26) means that the line $y_{1}(x)=x$ is tangent to the curve $y_{2}(x)=$ $\tanh (a x+b)$, consequently:

$$
\frac{d}{d x} \tanh (a x+b)=-a\left(\tanh ^{2}(b+a x)-1\right)=1
$$

With (31), the tangency condition becomes:

$$
a\left(1-x^{2}\right)=1 \rightarrow a=\frac{1}{1-x^{2}}
$$

and the solution of the problem is reduced to solving eq. (2) of [17], which can be written as:

$$
x=\tanh \left(\frac{x}{1-x^{2}}+b\right)=B_{1 / 2}\left(\frac{x}{1-x^{2}}+b\right)
$$

We can see that, actually, the problem involves only one parameter, $b$. The previous equation cannot be solved using generalized Lambert functions, as the argument of exponential is not linear, so the only one exact solution remains that of Siewert and Burkart, [17].

In [14], Siewert and Essig solved the Weiss equation of ferromagnetism:

$$
\zeta=\tanh \frac{1}{2}(j z \zeta+h)
$$

Alternative ways of solving, exactly or approximately, this equation were presented in [45], where the exact solution is written in terms of a Lambert generalized function with one upper and one lower parameter. The solution for the case $h=0$ was written as a generalized Lambert function $W(t ; s ; a)$ in [29], [30].

\section{Equations involving trigonometric and exponential functions}

In [22] and [23], the authors obtained the solutions of an equation basic to the theory of vibrating plates:

$$
a \tan x+\tanh x=0
$$

which appears also in quantum mechanics and electromagnetism. To find the real solutions of (37) means to find the real root of the exponential polynomial:

$$
\left(1+\frac{a}{2} i\right) e^{2 i x}-\left(1-\frac{a}{2} i\right) e^{2(1+i) x}+\left(1-\frac{a}{2} i\right) e^{2 x}-1-\frac{a}{2} i=0
$$

which is a generalized 3-upper and 3-lower complex parameter generalized Lambert function - a class of functions not studied yet.

However, we can made a certain progress in finding an approximate analytic solution of this equation using the algebraic approximation for $\tan x$ [46]:

$$
\tan x \simeq \frac{0.45 x}{1-\frac{2}{\pi} x}, 0<x<\frac{\pi}{2}
$$

This formula can be easily extended for any real $x[5]$. Replacing $\tan x$ in (37) according to (39), we get:

$$
e^{2 x} \frac{2 x-\frac{\pi}{\left(1-0.45 a \frac{\pi}{2}\right)}}{2 x-\frac{\pi}{\left(1+0.45 a \frac{\pi}{2}\right)}}=1
$$

so:

$$
x(a)=\frac{1}{2} W\left(\frac{\pi}{\left(1-0.45 a \frac{\pi}{2}\right)} ; \frac{\pi}{\left(1-0.45 a \frac{\pi}{2}\right)} ; 1\right)
$$

Eq. (40) is quite similar to the equation satisfied by the inverse Langevin function:

$$
e^{2 x}=\frac{A+1}{A-1} \frac{x+\frac{1}{A+1}}{x+\frac{1}{A-1}}
$$

with $x=L^{-1}(A)$, so the recipes for obtaining $L^{-1}$ could be useful also for an approximate evaluation of $W$ in (41).

As $\tanh x$ is, for $x \gtrsim 2$, a very slowly varying function, the roots of eq. (37) larger than $\pi / 2$ can be obtained, with reasonable accuracy, putting $\tanh x \simeq$ const on certain conveniently chosen intervals. For instance (see Figure 5, where the parameter $a$ from eq. (37) takes the value $a=1$ corresponding to the brown curve), the "exact" root $x_{1} \in(\pi, 3 \pi / 2)$ of eq. (37) is $x_{1}=3.9266$. If we approximate, for $x_{1}<x<3 \pi / 2$, the hyperbolic tangent as $\tanh x \simeq \tanh (3 \pi / 2)=0.999839$, eq.(37) becomes $\tan x=0.999839$, with the convenient root $x_{1 a}=$ 3.92691. The relative error of this approximation is about $-0.8 \cdot 10^{-4}$.

Applying the same method for $a=-1$ (corresponding to the green curve in Figure 3), we find for the root $x_{2}$ of eq. (37), $\pi / 2<x_{2}<\pi$, the approximate value $x_{2 a}=2.39938$, instead of the "exact" value, $x_{2}=2.36502$, so with an error of about $-1.5 \%$.

So, for the practitioner working in applied physics, in a domain where the experimental error is larger than the aforementioned ones, this crude and over-simplified approach gives acceptable results, for pragmatic reasons. 


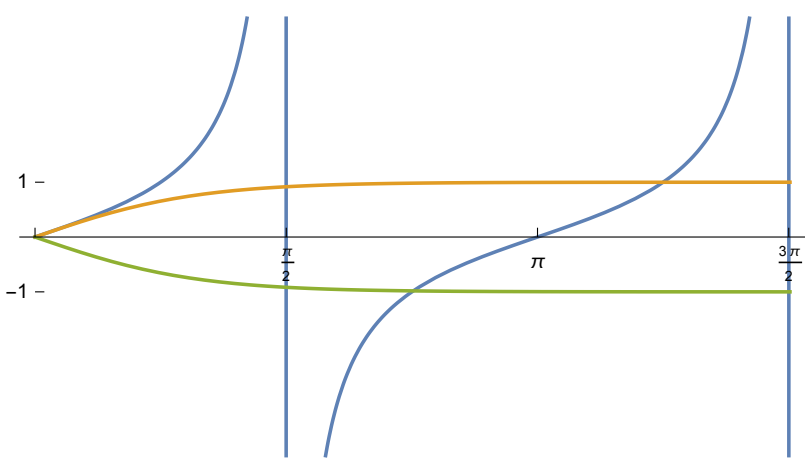

Figure 3: The plots of $\tan (\mathrm{x})$ (black), $\tanh (\mathrm{x})$ (green) and $-\tanh (\mathrm{x})$ (red).

The first root of the eq. (37) can be obtained for small values of $a$, after series expansions, as one of the roots of the algebraic equation:

$$
\frac{17}{315}(a-1) x^{6}+\frac{2}{15}(a+1) x^{4}+\frac{1}{3}(a-1) x^{2}+a+1=0
$$

which is a cubic equation in $z=x^{2}$. For instance, if $a=$ 0.1 , the error of the result obtained in this way is about $-4 \%\left(x_{\text {exact }}=1.2952, x_{\text {approx }}=1.3435\right)$. We can proceed similarly, when $a>>1$, with the equation:

$$
\tan x+\frac{1}{a} \tanh x=0
$$

\section{The Wright omega function}

The Wright omega $\omega(x)$ function appears in the asymptotic form of two equations solved by Siewert and his coworkers. It is related to the Lambert function by the identity [47]:

$$
W\left(e^{x}\right)=\omega(x)
$$

In [19], the authors solved a problem of elementary physics, obtaining "an exact analytical solution for the position-time relationship for an iverse-distance-squared force". Actually, they study the repulsive classical 1D movement of an electric charge in the field of another fixed charge. The repulsive force is given by the Coulomb law:

$$
m \frac{d^{2} r}{d t^{2}}=\frac{q Q}{4 \pi \varepsilon_{0} r^{2}}
$$

The initial condition is:

$$
t=0 \rightarrow \frac{d r}{d t}=0, r=r_{0}
$$

We shall describe the position of the moving charge by the dimensionless function $x(t)$, defined by:

$$
r(t)=r_{0} x(t)
$$

After two integrations of the equation of movement, we get the relation between position $x$ and time $t$ :

$$
\sqrt{x(x-1)}+\ln (\sqrt{x}+\sqrt{x-1})=\frac{t}{\tau}
$$

with $\tau$ given by:

$$
\tau=\sqrt{\frac{2 \pi \varepsilon_{0} m r_{0}^{3}}{q Q}}
$$

We shall study Siewert's result (48) at small and at large values of $t$. According to (48),

$$
x(0)=1
$$

so, for $t / \tau \ll 1$, we can put:

$$
x=1+X, \quad X<1
$$

and (49) can be approximated by:

$$
\ln \left(1+\frac{X}{2}+\sqrt{X}\right)=\frac{t}{\tau}-\sqrt{X}\left(1+\frac{X}{2}\right)
$$

and again, neglecting $X$ with respect to $\sqrt{X}$ :

$$
\ln (1+\sqrt{X})=\frac{t}{\tau}-\sqrt{X}
$$

or:

$$
2 \sqrt{X}=\frac{t}{\tau}
$$

and finally:

$$
X=\frac{1}{4 \tau^{2}} t^{2}
$$

At very small times, $t \ll \tau$, the movement is uniformly accelerated, as expected.

Asymptotically, $x \gg 1$ and (49) gives:

$$
\ln (2 \sqrt{x})=\frac{t}{\tau}-x
$$

or:

$$
2 x e^{2 x}=\exp \left(\frac{2 t}{\tau}-\ln 2\right) \rightarrow 2 x=W\left(\exp \left(\frac{2 t}{\tau}-\ln 2\right)\right)
$$

Consequently:

$$
x=\frac{1}{2} W\left(\exp \left(\frac{2 t}{\tau}-\ln 2\right)\right)
$$


where $W$ is the Lambert function. With 45, the asymptotic formula (58) can be written equivalently as:

$$
x(t)=\frac{1}{2} \omega\left(\frac{2 t}{\tau}-\ln 2\right), t>>
$$

The asymptotic expansion of the Lambert function is:

$$
W(x)=\ln x-\ln (\ln x)+\frac{\ln (\ln x)}{\ln x}+\ldots
$$

Keeping only the first term, the asymptotic formula (58) gives:

$$
x(t)=\frac{t}{\tau}-\frac{\ln 2}{2} \simeq \frac{t}{\tau}
$$

This is also an intuitive result, as, at very large distances, the repulsive force produced by the fixed charge becomes negligible small, and the movement becomes almost uniform. So, we checked that the movement starts by being uniformly accelerated and ends by being uniform.

In [10], Siewert and Burniston solved the Kepler equation for hyperbolic orbits:

$$
e \sinh F=F+N, \quad N>0
$$

whose solution cannot be reduced to generalized Lambert function. Asymptotically, $\sinh F \rightarrow \frac{1}{2} \exp F$ and (62) becomes:

$$
\frac{e}{2} \exp F=F+N, \quad N>0
$$

or, with $\exp F=f, F=\ln f$ :

$$
\frac{e}{2} f=\ln f+N
$$

so, a Wright equation, whose standard form is [47]:

$$
y+\ln y=z
$$

An interesting discussion about Kepler equation for both hyperbolic and elliptic (see Section 8) orbits is given in [51].

\section{Lambert function and generalized Lambert functions}

In [16], Siewert and Burniston found the solution of the equation:

$$
z e^{z}=a
$$

i.e. obtain an expression for the $W$ Lambert function, $a$ being a complex parameter [50]. In [12], Siewert solves "the familiar critical equation, described by age-diffusion theory, for a bare nuclear reactor":

$$
\frac{k \exp \left(-B^{2} \tau\right)}{1+B^{2} L^{2}}=1
$$

for $B^{2}$ (the buckling).

In [18], the author solves a more complicated equation:

$$
e^{z} \frac{z}{z+b}=a
$$

with $a$ - a complex parameter. So, he obtains an expression for the function $W(0 ;-b ; a)$, which can be written, at its turn, in terms of the Mezö - Baricz function $W_{r}$. For $a$ - real, the author refers to a paper of Wright [52].

\section{Transcendental equations involving trigonometric and algebraic functions}

In [15], the authors studied "the critical condition for a spherical reactor, described by elementary diffusion theory, surrounded by an infinite reflector":

$$
x \cot x=1-a-b x
$$

This equation can be written as:

$$
e^{2 i x} \frac{(b+2 i) x+a-1}{(b-2 i) x+a-1}=1
$$

so its solution is a generalized Lambert function of imaginary argument, with one upper and one lower complex parameter. This class of functions was recently studied by Mezö [28].

We can easily obtain an approximate analytical solution of (69), using the algebraic approximation of the tangent [46], [5]. We get, in this way, instead of (69), an approximate equation:

$$
x=\frac{0.45 \pi(x-n \pi)}{2 x-(2 n-1) \pi}(1-a-b x)
$$

which can be reduced to a second degree algebraic equation:

$x^{2}(1.4137 b+2)-3.1416 x(2 n-0.45 a+1.4137 b n-0.55)-$

$$
-4.4413 n(a-1)=0
$$

For $a=1, b=1, n=2$, the root of this equation is $x_{\text {approx }}=5.3628$, and the root of $(69)$ is $x_{\text {exact }}=5.49779$, so the error is about $2.5 \%$. 
In [13], Burniston and Siewert solved the equation:

$$
a \sin \zeta=\zeta
$$

which appears in quantum mechanics (defining the eigenenergies of a particle in a square well potential), in electromagnetism, in elasticity, in optics etc. Somewhat later, Siewert obtains a simpler solution [21]. A very precise approximate analytic solution of (73) was obtained through algebraization [2-5]; it is useful, for instance, for the calculation of energy levels in heterojunctions and quantum dots.

A more complicated variant of (73) is the Kepler equation for elliptic orbits [10] (the eccentricity, usually noted by $e$, will be written as $\varepsilon$, in order to avoid the confusion with the basis of Nepperian logarithms):

$$
\varepsilon \sin E=E-M, 0<\varepsilon<1,0<M<2 \pi
$$

We can obtain a quite precise solution of (74) approximating the first half-bump of sin by a cubic polynomial:

$$
y(x)=a x^{3}+b x^{2}+x
$$

where the coefficients $a, b$ can be determined by imposing the conditions:

$$
y\left(\frac{\pi}{2}\right)=1, y^{\prime}\left(\frac{\pi}{2}\right)=0
$$

We find:

$$
y(x)=\frac{4}{\pi^{3}}(\pi-4) x^{3}-\frac{4}{\pi^{2}}(\pi-3) x^{2}+x
$$

which fits quite well the function $\sin x$, for $0<x<\pi / 2$, as we can see in Figure 4.

If $e=0.9, M=0.1$, replacing $\sin x$ with the polynomial (77) in the Kepler equation (74), we obtain the solution $x_{1 p}=0.59955$, while the "exact" solution is $x_{1 e}=$ 0.59955 ; so, the error is $\varepsilon_{1} \simeq 5 \times 10^{-2}$. If $e=0.9$ and $M=0.5$, the polynomial approximation gives $x_{2 p}=1$. 3821 , and the "exact" solution is $x_{2 e}=1.38441$, so the error is $\varepsilon_{2} \simeq 1.7 \times 10^{-3}$. The plots in Fig. 4 show, intuitively, why the second approximation is more precise.

\section{Conclusions}

This paper is essentially focused on the transcendental equations studied by Siewert and his coworkers, considered in conjunction with the results obtained recently in the theory of generalized Lambert functions. Siewert's exact (and very complicated) results are replaced, whenever

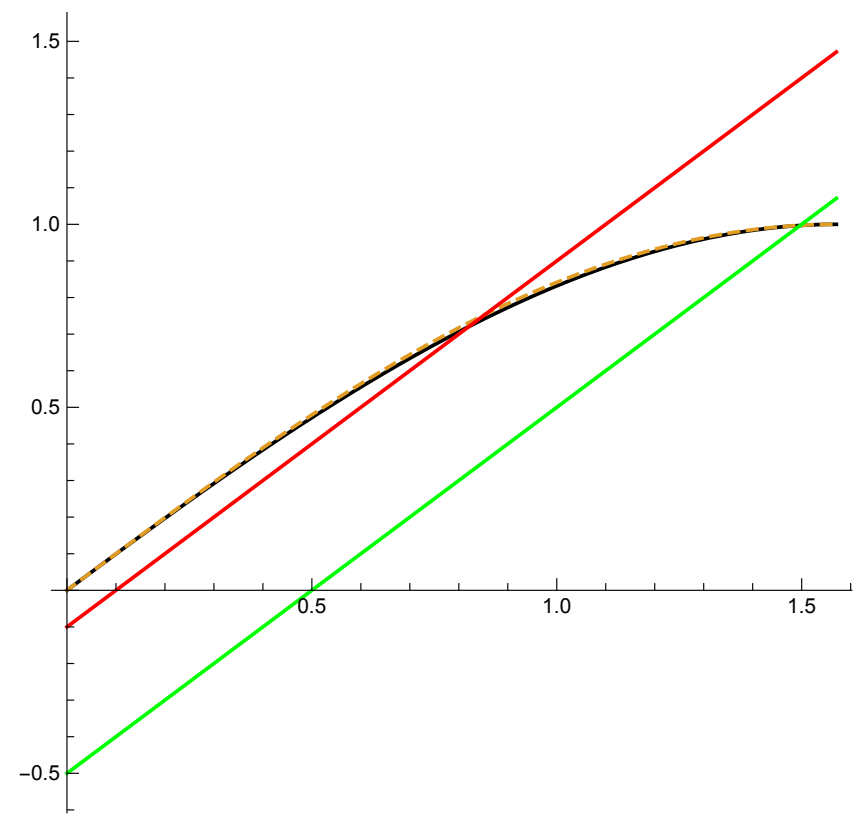

Figure 4: The plots of $y(x)$, eq. (77), black; $\sin (x)$, dashed; $x$ - 0.1, red; $x-0.5$, green.

possible, with the approximate analytical solutions of the same equations, obtained with some simple techniques. Some other results of Siewert and his coworkers, not connected to the generalized Lambert functions, are also discussed; in two cases, the asymptotic behavior of Siewert's solutions are expressed in terms of Wright $\omega$ function.

As sometimes the approximate expressions of the generalized Lambert functions are very precise (and their exact expressions are difficult to obtain), these approximations could provide a useful guidance of their exact behavior. Also, the "algebraization" of the transcendental equations (i.e. the replacement of the trigonometric functions with their various algebraic approximations) can provide, sometimes, surprisingly precise analytic solutions. They can be successfully used in applied physics or in the elementary presentation of advanced problems.

Acknowledgement: The author acknowledges the financial support of the IFIN-HH - ANCSI project PN 164201 01/2016 and to the IFIN-HH - JINR Dubna grant 04-4-11212015/17

\section{References}

[1] Griffiths D.J., Introduction to quantum mechanics, Prentice Hall, Upper Saddle River, New Jersey, 1995 
[2] Barsan V., Dragomir R., A new approximation for the square well problem, Optoel. Adv. Mat. - Rapid Commun., 2012, 6, 917-925.

[3] Barsan V., A new analytic approximation for the energy eigenvalues of a finite square well, Rom. Rep. Phys., 2012, 64, 685-694.

[4] Barsan V., Square wells, quantum wells and ultra-thin metallic films, Philos. Mag., 2014, 93, 1604-1617.

[5] Barsan V., Algebraic approximations for transcendental equations with applications in nanophysics, Philos. Mag., 2015, 95, 3023-3038.

[6] Marin M., On weak solutions in elasticity of dipolar bodies with voids, J. Comp. Appl. Math., 1997, 82, 291-297.

[7] Marin M., Harmonic vibrations in thermoelasticity of microstretch materials, J. Vibr. Acoust., ASME, 2010, 132, 0445011-044501-6.

[8] Sharma K., Marin M., Effect of distinct conductive and thermodynamic temperatures on the reflection of plane waves in micropolar elastic half-space, U.P.B. Sci. Bull., Series A-Appl. Math. Phys., 2013, 75, 121-132.

[9] Barsan V., New applications of the Lambert and generalized Lambert functions to ferromagnetism and quantum mechanics, arXiv:1611.01014v2, 2016.

[10] Siewert C.E., Burniston E.E., An exact analytical solution of Kepler's equation, Celestial Mechanics, 1972, 6, 294-304.

[11] Burniston E.E., Siewert C.E., The use of Riemann problems in solving a class of transcendental equations, Proc. Camb. Phil. Soc., 1973, 73, 111-118.

[12] Siewert C.E., An Exact Analytical Solution of an Elementary Critical Condition, Nuclear Sci. Eng., 1973, 51, 78-79.

[13] Burniston E.E., Siewert C.E., Exact analytical solution of the transcendental equation $a \sin \zeta=\zeta$, SIAM J. Appl. Math., 1973, 4, 460-465.

[14] Siewert C.E., Essig C. ., An Exact solution of a molecular Field Equation in the Theory of Ferromagnetism, J. Appl. Math. Phys., vol. 24, p. 281-286.

[15] Siewert C.E., Burniston E.E., On a critical condition, Nuclear Sci. Eng., 1973, 52, 150-151.

[16] Siewert C.E., Burniston E.E., Exact analytical solutions of $z e^{z}=$ a, J. Math. Anal. Applic., 1973, 43, 626-632.

[17] Siewert C.E., Burkart A.R., On Double Zeros of $x=\tanh (a x+b)$, J. Appl. Math., 1973, 24, 435-439.

[18] Siewert C.E., Solutions of the equation $z e^{z}=a(z+b)$, J. Math. Anal. Applic., 1974, 46, 329-337.

[19] Siewert C.E., Burniston E.E., An exact analytical solution for the position-time relationship for an iverse-distance-squared force, Int. J. Eng. Sci., 1974, 12, 861-863.

[20] Siewert C.E., Burniston E.E., An exact analytical solution of $x \operatorname{coth} x=\alpha x^{2}+1$, J. Comp. Appl. Math., 1976, 2, 19-26.

[21] Siewert C.E., Explicit results for the quantum-mechanical energy states basic to a finite square-well potential, J. Math. Phys., 1978, 19, 434-435.

[22] Siewert C.E., Phelps J.S. III, On solutions of a transcendental equation basic to the theory of vibrating plates, 1979, SIAM J. Math. Anal. 10, p. 105-108.

[23] Siewert C.E., Phelps J.S. III, Explicit solutions of $a \tan (\xi-k \pi)+$ $\tanh \xi=0$, J. Comp. Appl. Math., 1979, 5, 99-103.

[24] http://www4.ncsu.edu/ ces/publist.html

[25] Muskhelishvili N.l., Singular integral equations, Noordhoff, Groningen, 1953

[26] Mezö I., Baricz A., On the generalization of the Lambert W function, arXiv: 1408.3999v2, 2014.
[27] Mugnaini G., Generalization of Lambert W-function, Bessel polynomial and transcendental equations, arXiv:1501.00138v2, 2015.

[28] Mezo I., On the structure of the solution set of a generalized Euler-Lambert equation, J. Math. Anal. Appl., 2017, 455, 538553.

[29] Mezö I., G. Keady, Some physical applications of generalized Lambert functions, Eur. J. Phys., 2016, 37, 065802

[30] Mezö I., G. Keady, Some physical applications of generalized Lambert functions, Eur. J. Phys., 2016, 37, 37, 065802.

[31] Vonsovsky S. V., Magnetism, Wiley, New York, 1974

[32] https://en.wikipedia.org/wiki/Superparamagnetism

[33] Kuncser V. et al., Engineering Magnetic Properties of Nanostructures via Size Effects and Interphase Interaction, in: V. Kuncser, L. Miu (Eds.): Size effects in Nanostructures, Springer Series in Materials Science, Heidelberg, 2016

[34] Johal A.S., Dunstan D.J., Energy functions for rubber from microscopic potentials, J. Appl. Phys., 2007, 101, 084917

[35] Kröger M., Simple, admissible, and accurate approximants of the inverse Langevin and Brillouin functions, relevant for strong polymer deformations and flows, J. Non-Newtonian Fluid Mech., 2015, 223, 77-87.

[36] Jedynak R., Approximation of the inverse Langevin function revisited, Rheol. Acta, 2015, 54, 29.

[37] Petrosyan R., Improved approximations for some polymer extension models, 2017, Rheol. Acta, 56, 21-26.

[38] Kubo R., Statistical Mechanics, Elsevier, Amsterdam, 1965, Ch. 2, p. 135 and 157.

[39] Keady G., The Langevin function and truncated exponential distributions, arXiv:1501.02535v1

[40] Suehrcke H., McCormick P.G., An approximation for of the fractional time distribution of daily clearness index, J. Solar Energy, 1987, 39, 369-370.

[41] Arrott A.S., Approximations to Brillouin functions for analytic descriptions of ferromagnetism, J. Appl. Phys., 2008, 103, $07 \mathrm{C} 715$

[42] Stanley H. E., Introduction to phase transitions and critical phenomena, Oxford University Press, Oxford, 1971

[43] Kittel Ch., Introduction to solid state physics, 8th ed., Wiley, New York, 8th ed., 2005

[44] Kochmanski M., Paszkiewicz T., Wolski S., Eur. J. Phys., 2013, 34, 1555.

[45] Barsan V., Kuncser V., Exact and approximate analytical solutions of Weiss equation of ferromagnetism and their experimental relevance, Philos. Mag. Lett., 2017, 97, 359-371.

[46] de Alcantara Bonfim O.F., Griffiths D.J., Exact and approximate energy spectrum for the finite square well and related potentials, Am. J. Phys., 2006, 74, 43-47.7

[47] Corless R.M., Jeffrey D.J., On the Wright $\omega$ function, http://www.orcca.on.ca/TechReports/2000/TR-00-12.pdf (2000)

[48] Golmankhaneh A.K., Khatuni T., Porghoveh N.A., Baleanu D., Comparison of iterative methods by solving nonlinear SturmLiouville, Burgers and Navier-Stokes equation, Cent. Eur. J. Phys., 2012, 10, 966-976.

[49] Golmankhaneh A.K., Baleanu D., On nonlinear Klein - Gordon equation, Signal Processing, 2011, 91, 446-451.

[50] Corless R.M., Gonnet G.H., Hare D., Jeffrey D.J., Knuth D.E., Adv. Comput. Math.,1996, 5, 329-359. 
[51] https://en.wikipedia.org/wiki/Kepler\%27s_equation\#cite_ note-7
[52] Wright E.M., Stability criteria and the real roots of a transcendental equation, SIAM J. Appl. Math., 1961, 9, 136-148. 